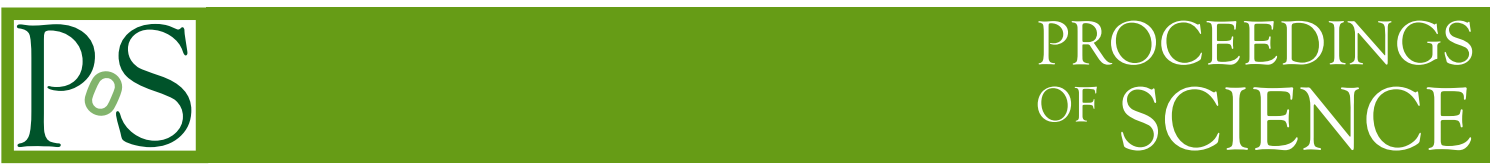

\title{
Definition and performance of muon physics object at CMS
}

Daniele TROCINO* on behalf of the CMS Collaboration ${ }^{\dagger}$

Northeastern University, Boston (USA)

E-mail: daniele.trocinoecern.ch

The performance of muon reconstruction and identification in CMS has been studied on data collected in pp collisions at $\sqrt{s}=7 \mathrm{TeV}$ and $8 \mathrm{TeV}$ at the LHC. We present measurements of muon reconstruction and trigger efficiencies, fake rates, and momentum scale and resolution.

The European Physical Society Conference on High Energy Physics -EPS-HEP2013

18-24 July 2013

Stockholm, Sweden

\footnotetext{
* Speaker.

${ }^{\dagger}$ The author would like to thank the CMS Muon Physics conveners, Silvia Goy López and Giovanni Abbiendi, for their active participation in preparing these proceedings and the related poster.
} 


\section{Introduction}

The Compact Muon Solenoid (CMS) [1] is a general-purpose detector at the Large Hadron Collider (LHC). Its physics program ranges from standard model presision measurements to Higgs and new physics searches. Muons are a distinctive signature for many of these processes. Thus CMS was designed for muon detection on a large momentum range, from few $\mathrm{GeV}$ up to the $\mathrm{TeV}$ scale. We present measurements of muon reconstruction and trigger efficiencies, misidentification rate, and momentum scale and resolution [2].

\section{Muon Reconstruction and Identification}

In the standard CMS reconstruction for pp collisions, tracks are first reconstructed independently in the inner silicon tracker (tracker tracks) and in the muon spectrometer (standalone-muon tracks), using a Kalman-filter technique. Starting from these tracks, two algorithms are used. In the global muon reconstruction, standalone and tracker tracks are extrapolated onto a common surface, taking into account the magnetic field and the expected energy loss and multiple scattering in the detector material, their parameters are compared, and if a matching pair of tracks is found, their hits are fitted to form a combined ( $\mathrm{global}$ ) track. In the tracker muon reconstruction, all tracker tracks are extrapolated to the muon system and matched to segments reconstructed in muon detectors.

We study the performance of two basic muon identification algorithms: the soft muon selection, which requires the candidate to be a tracker muon with tight requirements on the matched muon segment; and the tight muon selection, which requires the candidate to be both a global and a tracker muon, with stringent selections on the tracks' $\chi^{2}$, hit multiplicity, and transverse impact parameter. Figure 1 shows the distribution of muon transverse momentum $p_{\mathrm{T}}$, multiplied by the muon charge $q$, for an inclusive sample of soft muons collected with a zero-bias trigger (left), and the $p_{\mathrm{T}}$ spectrum of tight muons collected with a single-muon trigger with $p_{\mathrm{T}}$ threshold of $15 \mathrm{GeV} / \mathrm{c}$ (right). Data from $2010 \mathrm{LHC}$ run at $7 \mathrm{TeV}$ centre-of-mass energy are compared with simulations of multi-jet, quarkonia, $\mathrm{W}$ and $\mathrm{Z}$ bosons, non-resonant Drell-Yan, and top-pair production. The data agree with the predictions within $10 \%$ or better.
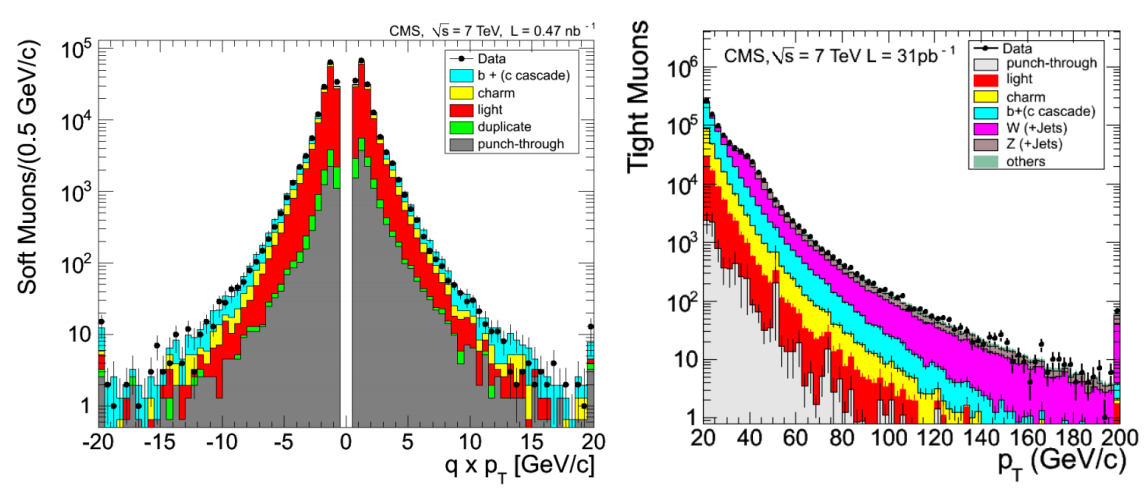

Figure 1: Data-simulation comparison, with data from $2010 \mathrm{LHC}$ run at $7 \mathrm{TeV}$ centre-of-mass energy. Left: charge $\times p_{\mathrm{T}}$ spectrum of soft muons collected with a zero-bias trigger. Right: $p_{\mathrm{T}}$ spectrum of tight muons collected with a single-muon trigger with $p_{\mathrm{T}}$ threshold of $15 \mathrm{GeV} / \mathrm{c}$. 


\section{Efficiency and Misidentification}

Muon identification and trigger efficiencies are measured with the tag-and-probe technique, using muons from selected $\mathrm{Z}$ decays in data and simulation [2]. Figure 2 shows the efficiency versus muon pseudorapity $\eta$ at $8 \mathrm{TeV}$ of the tight muon selection (left) and of a single-muon trigger with $p_{\mathrm{T}}$ threshold of $40 \mathrm{GeV} / \mathrm{c}$ with respect to tightly-selected muons (right). Tight muon efficiency is generally above $95 \%$ in the muon spectrometer barrel $(|\eta|<1.2)$ and $92 \%$ in the endcaps $(1.2<|\eta|<2.4)$, and is reproduced in simulation to within $1 \%$. Some data-simulation discrepancies in the trigger efficiency are due to the continuous evolution of triggers during the data taking, and are corrected with scale factors in physics analyses.
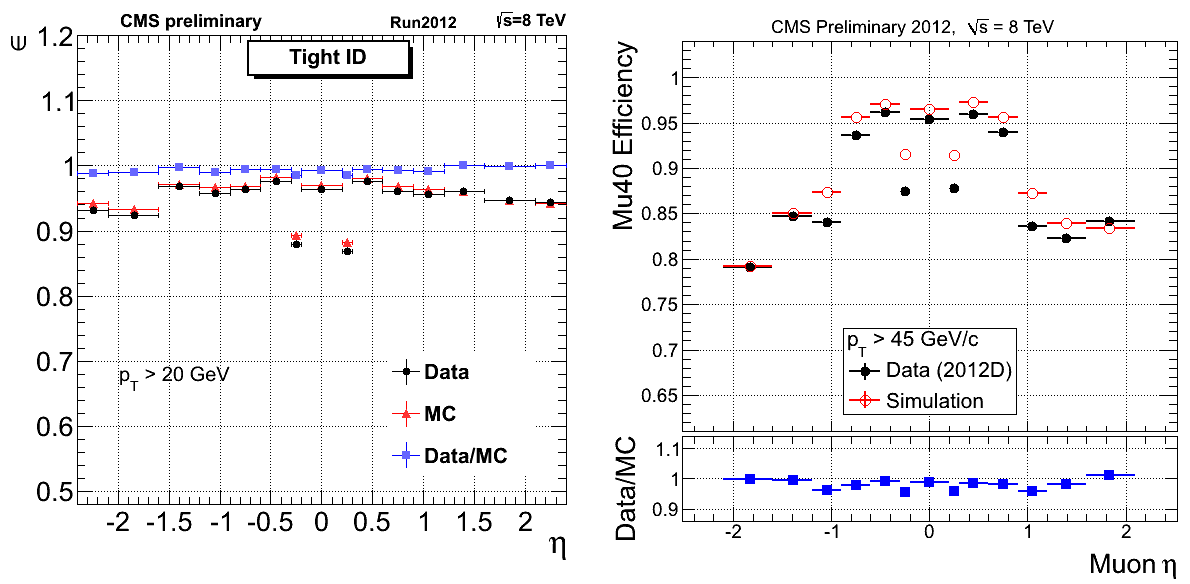

Figure 2: Efficiency of tight muon selection (left) and single-muon trigger with $p_{\mathrm{T}}>40 \mathrm{GeV} / \mathrm{c}$ (left) versus muon $\eta$, in selected $\mathrm{Z} \rightarrow \mu \mu$ events in $8 \mathrm{TeV}$ data (black) and simulation (red), and their ratio (blue).

Using the tag-and-probe method with decays $\phi \rightarrow \pi \pi, \Lambda \rightarrow \mathrm{p} \pi$, and $\mathrm{K}_{\mathrm{S}} \rightarrow \phi \phi$, hadron-tomuon misidentification rates are measured for pions, kaons, and protons, and found to be lower than $1 \%$ for the soft-muon selection, and lower than $0.1 \%$ for the tight muon selection.

\section{Muon Momentum Scale and Resolution}

The momentum scale and resolution of muons are studied using different approaches. For $p_{\mathrm{T}}$ values up to $100 \mathrm{GeV} / \mathrm{c}$, methods exploiting the $\mathrm{J} / \psi$ and $\mathrm{Z}$ mass resonances are used. The average bias in the muon momentum scale was measured with a precision of better than $0.2 \%$ and was found to be consistent with zero. The relative $p_{\mathrm{T}}$ resolution is $1.3-2.0 \%$ for muons in the barrel and better than $6 \%$ in the endcaps. For muons with $p_{\mathrm{T}}$ higher than $100 \mathrm{GeV} / \mathrm{c}$, the resolution is measured with cosmic rays in the barrel region, and is found to be better than $10 \%$ up to $1 \mathrm{TeV}$ for global and tracker muons, and better than $6 \%$ for specific high- $p_{\mathrm{T}}$ recostruction algorithms [2].

\section{References}

[1] CMS Collaboration, JINST 3 (2008) S08004.

[2] CMS Collaboration, JINST 7 (2012) P10002. 